\title{
DEVELOPMENT AND MANAGEMENT OF EAST-WEST EUROPEAN EXTENDED ENTERPRISES
}

Claudia Dold, Paolo Mauro, Rita Nøstdal, Fabrizio Pierpaoli

IAT - Institut für Arbeitswissenschaft und Technologiemanagement

Nobelstraße, 12 - 70569 Stuttgart - GERMANY, claudia.dold@iao.fhg.de

ITIA-CNR - Istituto di Tecnologie Industriali e Automazione

Viale Lombardia, 20/a - 20131 Milano-ITALY, p.mauro@itia.cnr.it

Fraunhofer Institute Industrial Engineering

Nobelstraße, 12 - 70569 Stuttgart - GERMANY, rita.noestdal@iao.fhg.de

ITIA-CNR - Istituto di Tecnologie Industriali e Automazione Viale Lombardia, 20/a - 20131 Milano-ITALY, f.pierpaoli@itia.cnr.it

\begin{abstract}
This paper describes a case study based research on current and best practices for collaboration management in manufacturing extended enterprises in an East-West European context. Case study findings show characteristic development paths leading from a simple customer-supplier relationship to the extended enterprise as well as major challenges resulting from cultural, technological and organizational variety. Typical issues that need to be addressed at the different development stages are deduced and clustered in a management framework for the extended enterprises in this special set up. First and second hand case studies are transformed into a best practice guideline for management matters like collaborative strategy, process alignment, common project management, knowledge transfer, communication and cultural diversity. Best practices cover suggestions for collaborative business in product development, production and sales.
\end{abstract}

\section{RATIONALE}

In the last decades increasing global competition, rapidly changing customer requirements and continuous evolution of information and communication technologies are forcing major changes in internal and external configuration of manufacturing enterprises. As a consequence, the increasing need of improving competitiveness by accessing new knowledge and markets is steering companies to expand their conventional borders turning their organizational structures into "virtual" structures. The evolution from the traditional forms of involvement of external partners in own business processes to the Extended Enterprise (EE) paradigm implies the necessity of developing adequate methodologies and tools to manage this new business context.

The broad context for this paper is characterized by the fact that production and development processes are spread much more often over several countries or regions 
in the world than before (von Behr, 2000). This is among others due to the growing competition from newly industrialised countries, the partial saturation of markets in the old industrial nations, the economic integration of the European Union, the enlarged market potentials in central and eastern Europe as well as on the Asian and American continent. All these events have far-reaching effects on the organisation of the value chain.

In order to face the enormous challenges to overcome turbulence and dynamism of current global markets, many west European SMEs have been increasingly looking for outsourcing opportunities and supply from companies in the eastern parts of Europe. Hitherto many European SMEs have developed long term relations with suppliers from countries of the eastern part of Europe (Le Goff, 2002) and some of them closely collaborate on issues like joint product development or shared production. Such co-operations are often a main factor for the competitiveness of European manufacturing SMEs that is so far mainly based on the combination of excellence in technology and processes in western Europe and cost advantages in eastern Europe (Boër et al., 2002). As cost advantages are gradually disappearing, the competitive edge of these collaborations must be grounded on other factors.

Any interferences in the management of collaborative business processes among the partners hinder the creation of value all along the supply chain.

This paper describes a development and management framework for EEs in an east-west European context and shows best practises for important organisational, technical and cultural aspects.

\section{OBJECTIVES AND RESEARCH APPROACH}

The objective of the case study research was to identify typical development paths and management issues of EEs in the above described context. Based on the empirical findings a reference development path for the evolution of company relationships from traditional customer/supplier to the $\mathrm{EE}$ collaboration was developed. Furthermore a set of best practice guidelines and methodologies for management areas like collaborative strategy, process alignment, common project management, knowledge transfer, communication and cultural diversity were elaborated, based on an extensive literature analysis and the case study results. These have been structured in a management framework. A roadmap has been conceived that, by the adoption of the methods provided in the framework, leads to a smooth development and improvement of the EE. Referring to the context described at the top, a limited number of corresponding surveys and studies were identified ${ }^{1}$.

Within the research, 17 collaborative business relations in different environments and including companies from 8 European countries have been analysed; in particular, 9 cases collaborate in an east-west European setting, 15 cases comprise one or more SMEs, 7 cases have partners collaborating in more than one EE and 8 cases show a collaboration duration of more than 5 years. The case studies were performed over a period of 5 months through semi-structured expert interviews with all relevant functions or departments of the companies involved. Topics covered in the interviews include the strategic backbone of the collaborations, overall corporative business processes, collaboration mechanisms, structures and procedures for knowledge transfer as well as problems encountered during the collaboration. 
The case study research gives indications for type of collaborations and how the collaboration develops over time according to indicators like information sharing, collaboration areas, legal agreements and so on. For both dimensions, case study findings were used to develop a set of "typical" set ups.

\section{TYPES OF EXTENDED ENTERPRISES}

The performed analysis and identification of case studies from literature and from industry has led to the detection of some meta models of $\mathrm{EE}$ that deserve a further investigation. The classification of the meta model that we propose does not intend to be complete as such but succeeded in clustering the industrial cases in a manner to stress communalities of current practices even in different industrial sectors. These communalities were derived from different objects that can be addressed by an industrial partnership: infrastructural facilities, product/market, technology. Starting from these main features we can introduce the following four meta models.

\section{Facilities sharing EE}

The EE is composed by partners that share resources or infrastructure facilities. This is observed for partners that are located close to each other. EEs in this category will ideally have complementary products or services in shared or separate markets, but partners will act as single companies towards customers. This introduces additional complexity to the resource management due to the need of a balanced dispatching of the common resources with some potential problems of conflict solving. EEs in this class shows a close collaboration in early phases as agreements on modalities for cost splitting, use of resources, common investment planning etc. will have to be settled. Facilities sharing EEs may be market or product oriented.

\section{Market oriented EE}

This form of partnership is characterized by a more market centred cooperation, as in the case of similar or complementary end products for different markets that hinder to perceive the partner as a competitor. The rationale for this collaboration relies on the necessity to exploit synergies at commercial and distribution level and may develop into common product planning and development, shared and separate branding strategies for different markets and shared logistic facilities and mutual use of distribution networks in different regions. Major benefits can be achieved if the respective markets are geographically dispersed cause the cost and investment for setting up a new distribution network can be dramatically reduced. This market centred integration is well promising in term of consolidating the $\mathrm{EE}$, because it is open to the definition of a common strategy, that can imply enlargement of product range, common product development, market expansion strategy, etc.

\section{Product oriented EE}

The typical configuration of such EEs implies the involvement of original equipment manufacturing (OEM) and one or more of his key suppliers. It is often the evolution of long term relationship with critical component suppliers and aims at consolidating the relationship and building a sort of privileged channel of supply. This kind of collaboration is expected to bring benefits from both side: from the 
point of view of the OEM the collaboration implies the guarantee of on-time and conform supplies. On the other hand, the supplier has more confidence on the demand volume and times and receives direct sensible information that can help plan production in a more effective manner. The leitmotif of such an extended collaboration is trust that must be achieved at different level, all along the manufacturing cycle. Trust is definitely essential when the collaboration implies codevelopment of new products since knowledge transfer and confidentiality are leading issues at this stage of the collaboration. In the east-west collaborations that were analysed, this configuration is characterized by a twofold beneficial aspect: efficacious knowledge transfer from western to eastern partner with the stimulus to upgrade manufacturing equipment and high conformity low cost supplies.

\section{OEM-dominated EE}

This form of partnership can be interpreted as the outermost case of a product oriented EE. It is represented by those cases, such as belonging to automotive industry, where one partner is predominant over the others for dimensions and relevance. In this case the trust is often substituted by an imposition in which the leader has the strength to impose philosophy, rules and standards and partners have but to respect such rules. In this study, we do not consider these EEs, as the collaborative aspect is overruled by a hierarchical set-up ${ }^{2}$.

\section{DEVELOPMENT PATH TOWARDS THE EE}

Starting from the case studies a typical development path of EEs has been derived which defines the major steps of interaction between companies that are intended to tighten the collaboration practices from a traditional customer-supplier relationship to a full implementation of the EE paradigm. This evolution path has been defined in the most generic manner in order to be of generic validity. The steps of the evolution path are not mandatory for all the EEs and the entry point in the path can occur at each of the different stages depending on the current features of the collaboration or on the specific industry. The development path towards the Extended Enterprise consists of five stages: customer-supplier, integrated supply chain, advanced integrated supply chain, collaboration level, Extended Enterprise (see Figure 1).

The customer-supplier relationship emphasises product and product support and is representing the very basic way of relying on a partner to supply a product/service.

The integrated supply chain relationship is focused on the processes associated with the reduction of the total cost of the supply-chain, including, but not limited to, procurement, inventory management and product deployment. A totally integrated supply-chain enables an end-user to more effectively and cost-efficiently manage inventory and transaction costs.

The advanced integrated supply chain step includes collaborative forecasting and planning, joint planning of capacity use and inventory. It is the latest generation of very sophisticated supply chains.

At the collaboration level the collaboration becomes closer and takes form of a long term relationship. In this case the collaborating partners are sharing work in process and are performing collaborative value chain planning. The partners strive to 
adapt each others systems and processes and introduce standardisation as a way to make information exchange easier and effective.

The final Extended Enterprise stage is the goal of a long term collaboration activity. Obviously, it is more difficult to achieve a high level collaboration without proper trust and mutual understanding. Companies that collaborate at the final Extended Enterprise level share every critical activity, from joint long term strategic planning to joint operational management, adopting common project management rules and acquiring a complete understanding of the economic and political environment of the partner.

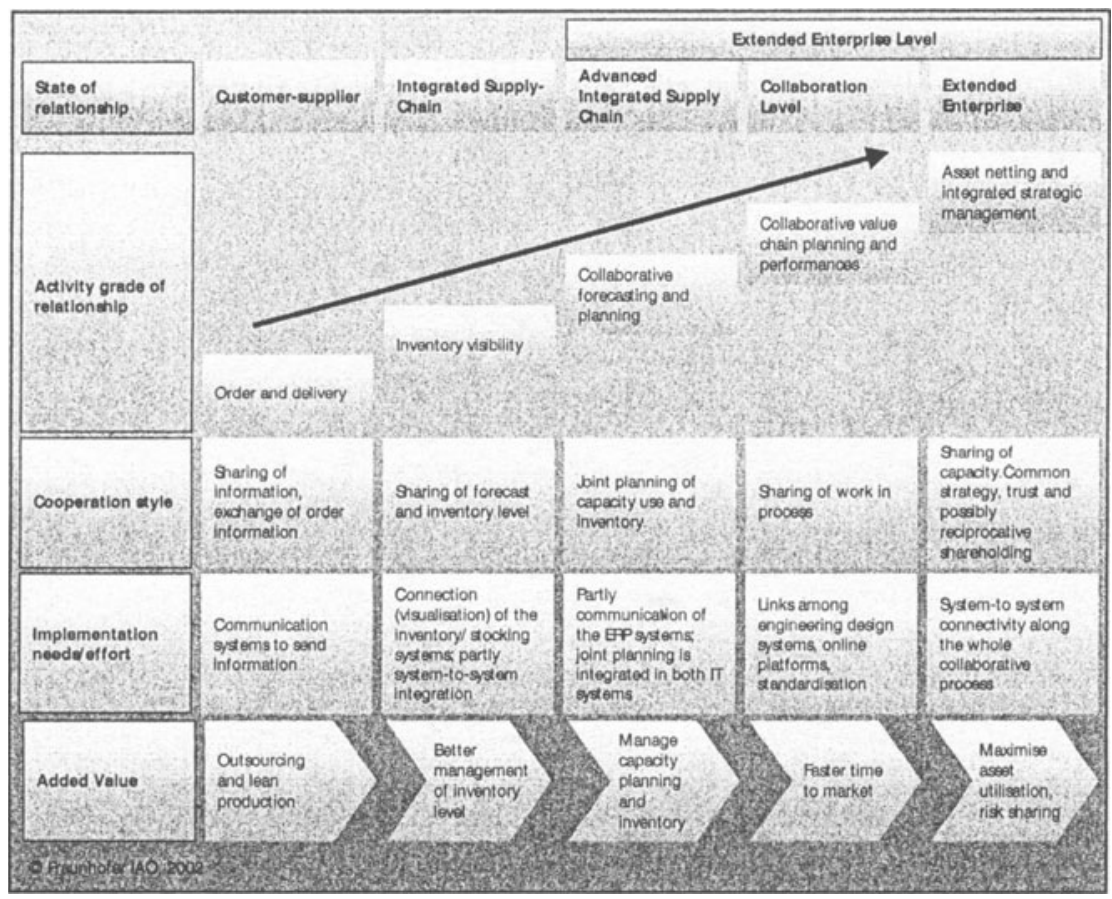

Figure 1 - The EE development path

Case studies show that in most product oriented collaborations, partners will typically enter at a "customer-supplier" level and develop towards an EE-level over a period of several years. Management issues are approached gradually and often not anticipated upfront. In a strong hierarchical setting this development is typically faster. Facilities sharing EEs are commonly entering at the level of Extended Enterprise in the sense of cooperation style and legal agreements, while management issues are handled similar as in product oriented EEs. Market oriented EEs tend also to enter at stage 1, but will earlier plan the development towards more close collaboration and identify clear development stages in early phases of the collaboration. 


\section{THE EE MANAGEMENT FRAMEWORK AND ROADMAP}

The analysis of the interviewed EEs highlighted typical problems for the management and operation of critical issues in different thematic areas. Management issues from different case studies are clustered and may be summarised as follows.

Integration strategy: the main finding in this concern is an overall lack of a common strategic approach and of a formal agreement that establishes the sharing of benefits and risks between partners.

Collaboration: under this respect language and cultural issues play a fundamental role: different languages and working cultures can hinder the daily activities. Difficulties in establishing a community spirit and in changing from a competitive attitude to a collaborative one.

Knowledge sharing: no knowledge management strategy is currently performed in any of the analysed case studies; this implies a dispersion of distinctive knowhow and competences and difficulties in the exchange of information (excess of paper based information flow).

Technology and Information Technology: in this area the necessity of bridging the gap in technical expertise, technological infrastructure and IT tools (software integration and compatibility) is the first need of each EE.

Organization: different standards in production and product development technology within an EE is a frequent source of inefficient processes and redundant work. Need for common working procedures and wide cross-organisational labour mobility aiming at assimilating common practices.

Logistics: lack in common planning and warehouses managing, difficulties in coordinating and tracking deliveries.

Project Management: a strong necessity of improving cooperation and project management processes has emerged, due to insufficient visibility on partner's activities and lack of inter-functional coordination.

In order to tackle the expressed needs a framework for the management of the $\mathrm{EE}$ has been conceived (see Table 1). The framework includes the main topics that need to be addressed in order to guarantee a smooth management of the EE; the framework can be seen as a cluster of methodologies that has been developed with the aim of accompanying companies along their evolution path towards the final Extended Enterprise stage.

The EE management Roadmap is a compilation of typical issues, best practises and development stages into a generic roadmap for the development and management of a EE. The methodologies embedded in the framework are explicitly intended to act as a concrete support during the roadmap execution. The methodical procedure consists of three main steps, which can be summarised (adapted from: Schmidt et al., 1996; Bea, Göbel, 1999) as follows:

1. Analysis of the current situation of the collaboration. Defining the current position on the development path.

2. Creation of a vision for the collaboration. Defining the desired way of working together in the future. Defining the desired position on the development path.

3. Definition of relevant steps to reach the desired position on the development path. Fulfil requirements for the desired level of collaborative activity.

Ad 1) In order to evaluate the EEs in reference to the development path described above, a method was developed that identifies gaps between the status quo 
and best practice. Seven benchmark areas were extracted, each containing of 5-8 descriptive sub-dimensions. The sub-dimensions are clearly representing the aspects of main interest and that of main criticality about an EE. Benchmark areas are collaboration, logistics, knowledge sharing, technology and IT, organization, integration strategy and project management. The result is a target table with areas, describing criteria and scales for each of the 5 stages of the development path.

Ad 2) The next step is to establish the EE vision with the help of target tables for each benchmark category. Following this approach, every analysed enterprise has its EE profile/classification in the development path and can now be evaluated concerning identification, definition and evaluation of coming fields of gaps/needs and first remedies through comparison with „Best in Class“ and „Best Practise”.

Ad 3) In order to build a successful extended enterprise, a roadmap has to be built, that refers to a sequence of steps that have to be pursued to reach the stage of an "Extended Enterprise" as described in the development path. The roadmap is embedded in the overall methodology for the building of an EE supporting the last step in the methodology - the action needed to implement management issues to fulfil the criteria of the desired position on the development path. It includes checklists and steps in every management area that have to be considered depending on the current situation of the collaboration. For every position in the development path, the management efforts are described and it can be derived what has to be implemented to fulfil the criteria of the next level on the development path. After reaching the Extended Enterprise Level (levels 3 to 5 on the development path) three defined milestones and checklists including goals reached, problems and gaps that still need to be filled, have to be processed. Once the desired goal has been achieved, the real collaborative activities need to be rechecked by using the development path and the illustrated roadmap and have perhaps to be repeated.

By using this compact procedure backed up with the different instruments and methods which were developed in the analysis, it becomes possible to build an EE starting from a simple customer-supplier relationship and going all the way to the final Extended Enterprise level. This practical approach can be generally applied to all collaboration relationships and business co-operations.

Table 1 - A management framework for the EE

\begin{tabular}{|c|c|c|}
\hline Organization & Collaboration & Logistics \\
\hline $\begin{array}{l}\text { A module for the implementation of } \\
\text { one coherent and common working } \\
\text { culture within the EE, including } \\
\text { strategic planning, project and } \\
\text { operational management }\end{array}$ & $\begin{array}{l}\text { A module for initiation and } \\
\text { establishment of the transfer } \\
\text { of knowledge, know how and } \\
\text { capabilities within the EE, to } \\
\text { let them grow together }\end{array}$ & $\begin{array}{l}\text { A module for the implementation of } \\
\text { an efficient logistic system between/ } \\
\text { within the EE, including control } \\
\text { systems, facility management and } \\
\text { transportation systems }\end{array}$ \\
\hline $\begin{array}{l}\text { Strategy: } \\
\text { Strategic planning for future goal, } \\
\text { decision of the direction of action }\end{array}$ & $\begin{array}{l}\text { Knowledge transfer: } \\
\text { Supports the exchange of } \\
\text { know how within the EE }\end{array}$ & $\begin{array}{l}\text { Control system: } \\
\text { Monitors logistics processes such as } \\
\text { storage or transportation systems }\end{array}$ \\
\hline $\begin{array}{l}\text { Project: } \\
\text { Efficient management of project } \\
\text { related work and project related } \\
\text { goals, of resources and capacity }\end{array}$ & $\begin{array}{l}\text { Culture: } \\
\text { Overcomes cultural } \\
\text { differences. EE should profit } \\
\text { form the cultural variety }\end{array}$ & $\begin{array}{l}\text { Facilities: } \\
\text { Management of the logistic facilities } \\
\text { to make sure that they support the } \\
\text { production process and time frames }\end{array}$ \\
\hline $\begin{array}{l}\text { Processes: } \\
\text { Management of the current needed } \\
\text { actions for running the business }\end{array}$ & $\begin{array}{l}\text { Communication: } \\
\text { Management of all } \\
\text { communication related topics } \\
\text { between the EE partners }\end{array}$ & $\begin{array}{l}\text { Transportation system: } \\
\text { Management of the transportation of } \\
\text { goods over often far distances } \\
\text { between the EE partners }\end{array}$ \\
\hline
\end{tabular}




\section{CONCLUSION}

The planned evolution of an extended enterprise is a complex management matter of several dimensions. An integrative approach for the management of these dimensions, based on a mutual and agreed development path towards a certain collaboration level, may support extended enterprises in identifying and exploring a common competitive edge. The basis for such a development is trust. Here we would like to quote from one case study: “...trust in the extended enterprise means predictability of behaviour and reactions in common issues. This can only be achieved within a win-win situation. This is the ground for any extended enterprise".

\section{Acknowledgements}

This work is based on and represents the ongoing activities in the European Union co-funded research project Prominence ${ }^{3}$.

We would like to thank the whole project Consortium for its support.

We would like to thank Dipl. Ing. Carsten Schaetzberger for his precious contribution in the drawing up of this paper.

\section{REFERENCES}

1. Bea FX, Göbel E. Organisation. Stuttgart, 1999.

2. Boër CR, Bosani R, Dold C, Mauro P, Nøstdal R, Pierpaoli F, Ristol Jorba S. Prominence ProjectPromoting Inter-European Networks of Collaborating Extended Enterprises. In Proceedings of the ACS'02 - SCM Conference, October 23-25, Poland, 2002.

3. Dathe, J. Kooperationen - Leitfaden für Unternehmen, Strategien, Erfahrungen und Grenzen in Europa. München, 1998.

4. Dyer, HD. Collaborative Advantage - Winning Through Extended Enterprise Supplier Networks. Oxford, 2000.

5. Edwards V, Lawrence P. Management in Eastern Europe. 2000.

6. Le Goff, Solenn. Prominence. Unpublished internal document on the EU co-funded project Prominence. 2002.

7. Schmidt HJ, Bullinger HJ, Warnecke HJ. Neue Organisationsformen im Unternehmen. Heidelberg, 1996.

8. The International Bank of Reconstruction and Development/The World Bank. Transition - The First Ten Years - Analysis and Lessons for Eastern Europe and the Former Soviet Union. 2002.

9. Von Behr, M. Produktion international: Beispiele aus der mittelständischen Industrie von der Standortwahl bis zum integrierten Netzwerk. Eschborn, RKW, 2000.

\footnotetext{
${ }^{1}$ For example: Dathe's (1998) research in the field of multinational project management among European countries shows that a appropriate implementing of teamwork is one of the most important key factors. Dyer (2000) shows the necessity of trust (reliability, fairness and goodwill) and knowledge transfer as one of the most important collaboration criteria and proves a correlation between the defects per vehicle in car manufacturing to the man-days of face-to-face contact.

${ }^{2}$ Although, it is important to note, that product oriented extended enterprises are often defined by a rather hierarchical set up. In the case of the OEM-dominated EEs, the hierarchy is predominant.

${ }^{3}$ Promoting Inter-European Networks of Collaborating Extended Enterprises. No.: GRD-2001-40747.
} 